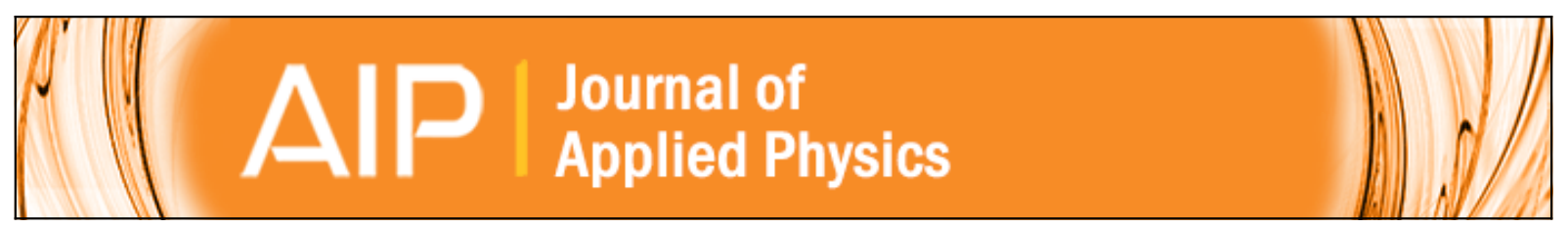

\title{
Cavity mode gain alignment in GaAsSb-based near-infrared vertical cavity lasers studied by spectroscopy and device measurements
}

G. Blume, K. Hild, I. P. Marko, T. J. C. Hosea, S.-Q. Yu, S. A. Chaparro, N. Samal, S. R. Johnson, Y.-H. Zhang, and S. J. Sweeney

Citation: Journal of Applied Physics 112, 033108 (2012); doi: 10.1063/1.4744985

View online: http://dx.doi.org/10.1063/1.4744985

View Table of Contents: http://scitation.aip.org/content/aip/journal/jap/112/3?ver=pdfcov

Published by the AIP Publishing

\section{Articles you may be interested in}

Detecting and tuning anisotropic mode splitting induced by birefringence in an InGaAs/GaAs/AIGaAs verticalcavity surface-emitting laser

J. Appl. Phys. 111, 043109 (2012); 10.1063/1.3682648

Optimal photonic-crystal parameters assuring single-mode operation of $1300 \mathrm{~nm}$ AllnGaAs vertical-cavity surface-emitting laser

J. Appl. Phys. 105, 093102 (2009); 10.1063/1.3115449

Self-sustained pulsation in the oxide-confined vertical-cavity surface-emitting lasers based on submonolayer InGaAs quantum dots

Appl. Phys. Lett. 91, 121106 (2007); 10.1063/1.2784937

Reverse-bias emission sheds light on the failure mechanism of degraded vertical-cavity surface-emitting lasers J. Appl. Phys. 99, 123113 (2006); 10.1063/1.2206852

Blue vertical-cavity surface-emitting lasers based on second-harmonic generation grown on (311)B and (411)A GaAs substrates

J. Appl. Phys. 87, 1597 (2000); 10.1063/1.372065

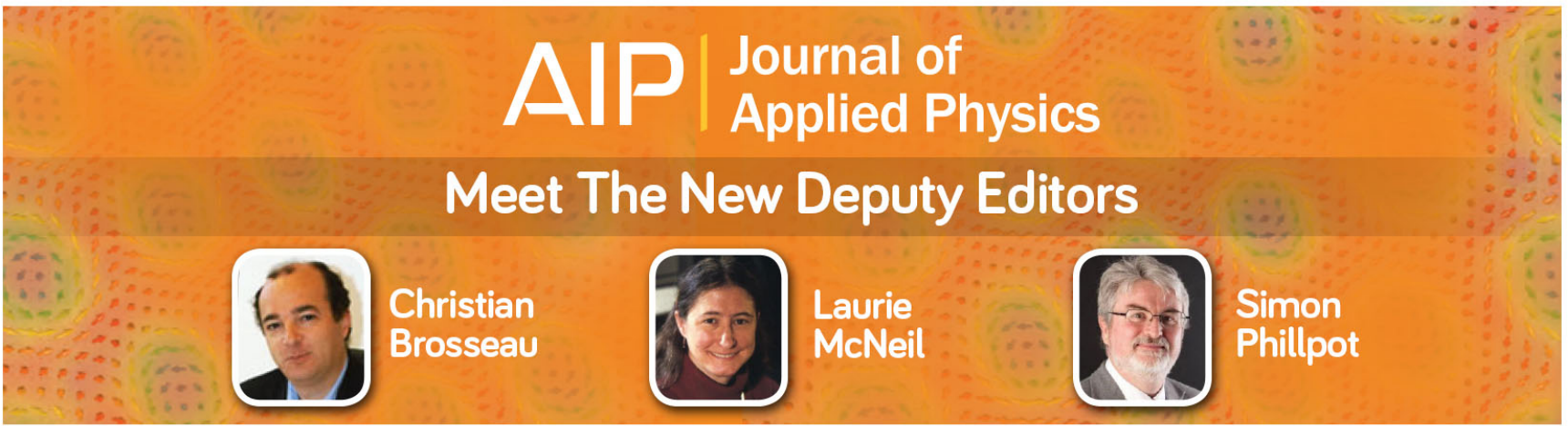




\title{
Cavity mode gain alignment in GaAsSb-based near-infrared vertical cavity lasers studied by spectroscopy and device measurements
}

\author{
G. Blume, ${ }^{1, a)}$ K. Hild, ${ }^{1}$ I. P. Marko, ${ }^{1}$ T. J. C. Hosea, ${ }^{1,2, b)}$ S.-Q. Yu, ${ }^{3, c)}$ S. A. Chaparro, ${ }^{3}$ \\ N. Samal, ${ }^{3}$ S. R. Johnson, ${ }^{3}$ Y.-H. Zhang, ${ }^{3}$ and S. J. Sweeney ${ }^{1, d)}$ \\ ${ }^{1}$ Advanced Technology Institute and Department of Physics, University of Surrey, Guildford, Surrey GU2 7XH, \\ United Kingdom \\ ${ }^{2}$ Ibnu Sina Institute, Universiti Teknologi Malaysia, 81310 UTM Skudai, Johor, Malaysia \\ ${ }^{3}$ School of Electrical, Computer and Energy Engineering and Center for Photonics Innovation, Arizona State \\ University, Tempe, Arizona 85287-5706, USA
}

(Received 21 March 2012; accepted 11 July 2012; published online 8 August 2012)

\begin{abstract}
We present a combination of spectroscopy and device measurements on GaAsSb/GaAs vertical-cavity surface-emitting laser (VCSEL) structures to determine the temperature at which the wavelength of the VCSEL cavity mode $(\mathrm{CM})$ aligns with that of the quantum well $(\mathrm{QW})$ ground-state transition (GST), and therefore the gain peak. We find that, despite the achievement of room temperature (RT) continuous wave lasing in VCSEL devices, the QW transition and the $\mathrm{CM}$ are actually slightly misaligned at this temperature; room temperature electroluminescence measurements from a cleaved edge of the VCSEL wafer indicate that the $300 \mathrm{~K}$ QW GST energy is at $0.975 \pm 0.005 \mathrm{eV}$, while the $\mathrm{CM}$ measured in the VCSEL surface reflectivity spectra is at $0.9805 \pm 0.0002 \mathrm{eV}$. When the wafer sample is cooled, the CM and QW GST can be brought into alignment at $270 \pm 10 \mathrm{~K}$, as confirmed by temperature-dependent electro-modulated reflectance (ER) and edge-electroluminescence spectroscopic studies. This alignment temperature is further confirmed by comparing the temperature dependence of the emission energy of a fabricated VCSEL device with that of an edge-emitting laser structure with a nominally identical active region. The study suggests that for further device improvement, the room temperature $\mathrm{CM}$ and $\mathrm{QW}$ GST energies should be more closely matched and both designed to a smaller energy of about $0.95 \mathrm{eV}$, somewhat closer to the $1.31 \mu \mathrm{m}$ target. The study amply demonstrates the usefulness of non-destructive ER characterisation techniques in VCSEL manufacturing with GaAsSb-based QWs. (C) 2012 American Institute of Physics. [http://dx.doi.org/10.1063/1.4744985]
\end{abstract}

\section{INTRODUCTION}

Vertical-cavity surface-emitting lasers (VCSELs) emitting close to $1.3 \mu \mathrm{m}$ are of considerable importance for the development of metropolitan area networks as they provide the necessary properties, such as low temperature dependence, low threshold currents, and circular beam profile, for future fibre-to-the-home applications for the $1.31 \mu \mathrm{m}$ zero dispersion wavelength of standard silica fibres. ${ }^{1}$ Traditionally, lasers operating at this wavelength have been based on InP. However, it is difficult to grow the high quality distributed Bragg reflectors (DBRs), which are needed for VCSELs, based on InP, and complex approaches, such as wafer-bonding, are required. ${ }^{2}$ Since DBRs at this wavelength are relatively easily grown on $\mathrm{GaAs}$ using $\mathrm{GaAs} / \mathrm{Al}(\mathrm{GaAs})$ layer pairs, considerable effort has also been devoted to the development of GaAs-based active regions which emit near $1.3 \mu \mathrm{m}$. Examples include highly strained $\mathrm{InGaAs}^{3}{ }^{3}$ InAs quantum dots, ${ }^{4}$ and GaInNAs-based quantum wells (QWs) all of which have been the subject of extensive research.

\footnotetext{
a) Present address: Ferdinand-Braun-Institut, Leibnitz-Institut für Höchstfrequenztechnik, Gustav-Kirchhoff-Straße 4, 12489 Berlin, Germany.

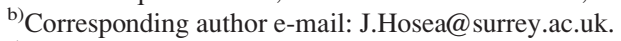

${ }^{c}$ Present address: Department of Electrical Engineering, University of Arkansas, 3217 Bell Engineering, Fayetteville, Arkansas 72701, USA.

d)e-mail: s.sweeney@surrey.ac.uk.
}

Laser operation has been demonstrated at $1.3 \mu \mathrm{m}$ using GaInNAs-based QWs, ${ }^{5}$ but their performance is limited by difficulties related to nitrogen incorporation, resulting in degraded material quality and lifetimes. Laser operation has also been demonstrated at $1.3 \mu \mathrm{m}$ with quantum dots, ${ }^{4}$ but such active layers have relatively low gain, requiring long cavity lengths for edge-emitting lasers (EELs), which is expensive in terms of wafer "real-estate." VCSELs based upon quantum dots suffer from the lack of gain, requiring a high number of layers, leading to carrier transport problems. An alternative approach, which we examine here, does not suffer from such disadvantages but has been less exhaustively studied. This is the use of GaAsSb QWs in VCSELs grown on GaAs substrates, ${ }^{6}$ to try to find a $1.3 \mu \mathrm{m}$ temperature insensitive emitter with good beam qualities using well-established GaAs technologies. It is possible to achieve GaAsSb-based QW emission near $1.3 \mu \mathrm{m}$, despite the considerable compressive strain due to mismatch with the GaAs substrate. It was initially found that the high compressive strain $(>\sim 2 \%)$ caused material quality problems when growing a second QW. Therefore, additional GaAsP layers were introduced, which have a smaller lattice constant than GaAs and partly compensate the strain. ${ }^{7-12}$ This enables one to grow multiple GaAsSb QWs thereby increasing the luminescence intensity.

As is well known, an important condition necessary for the efficient operation of a VCSEL structure is the alignment 
of the wavelengths of the cavity mode (CM) dip of the VCSEL reflectivity spectrum and the gain peak of the active QW region. It is generally accepted that the achievement of this alignment at the desired operating temperature should give close to ideal device operating conditions when other effects such as self-heating and non-radiative recombination have been taken into account. A small change in this alignment can have a considerable effect on the threshold current and output power. ${ }^{13}$ Here, we undertake a detailed spectroscopic investigation of the temperature dependence of the relative alignment of the $\mathrm{CM}$ dip with the $\mathrm{QW}$ ground-state transition (GST) energy (which largely dictates the peak energy in the gain spectrum due to the active QW region) of a GaAsSb/GaAs VCSEL structure, and corroborate these with spectroscopic measurements of the temperature dependence of the emission wavelengths of processed devices.

\section{SAMPLE AND EXPERIMENTAL DETAILS AND INITIAL DEVICE RESULTS}

The VCSEL structure studied here was grown by solid source molecular beam epitaxy on an n-doped GaAs substrate (doped to $\sim 2 \times 10^{17} \mathrm{~cm}^{-3}$ ). It has top and bottom stacks of $\mathrm{Al}_{0.91} \mathrm{Ga}_{0.09} \mathrm{As} / \mathrm{GaAs}$ DBR mirrors; an $\mathrm{n}$-doped bottom DBR with 35 pairs and a p-doped top DBR with 25 pairs. Low doping levels of 5 to $10 \times 10^{17} \mathrm{~cm}^{-3}$ were intentionally used throughout the entire mirrors in order to minimise free-carrier absorption. The mirror stacks enclose an $\mathrm{Al}_{0.25} \mathrm{Ga}_{0.75} \mathrm{As}$ cavity region with an optical thickness equal to the targeted room temperature (RT) free space emission wavelength $(\lambda)$ of $1.26 \mu \mathrm{m}(0.984 \mathrm{eV})$. Three QW structures are centred near the central anti-node of the cavity standing wave. Such $1 \times \lambda$ symmetric cavities with a single central anti-node in the cavity are typically used in order to obtain efficient symmetric electrical injection efficiency. Each of the three QW structures consists of a central $\mathrm{GaAs}_{0.65} \mathrm{Sb}_{0.35}$ QW of thickness $7 \mathrm{~nm}$, sandwiched by two GaAs barriers of thickness $3 \mathrm{~nm}$, and further sandwiched by two $8 \mathrm{~nm}$-thick $\mathrm{GaAs}_{0.8} \mathrm{P}_{0.2}$ strain-compensation layers. For further sample details see Dowd et al. ${ }^{10}$ For the spectroscopic investigations, the structure was capped with a $25 \mathrm{~nm}$ layer of $\mathrm{In}_{0.5} \mathrm{Ga}_{0.5} \mathrm{P}$ (i.e., close to lattice-matched) on top of the GaAs to protect the GaAs surface during processing, and a $5 \mathrm{~nm}$ semi-transparent Pt film evaporated on top of a piece of the VCSEL wafer to serve as an electrical contact.

Devices were also fabricated from the same VCSEL wafer, with different oxide aperture and optical window diameters. $^{7,14}$ It was found that continuous wave (CW) lasing operation could be obtained at RT for a VCSEL device with a $9 \mu \mathrm{m}$ diameter aperture with threshold currents above approximately $1 \mathrm{~mA}$ (current density of $\sim 1.5 \mathrm{kA} / \mathrm{cm}$.) As described later, pulsed operation was achieved at RT at a current density of $1.2 \mathrm{kA} / \mathrm{cm}^{2}$ for both $9 \mu \mathrm{m}$ and $27 \mu \mathrm{m}$ diameter devices. For these device studies, an edge-emitting laser device with a nominally identical active region to that of the VCSEL was also grown and fabricated.

A closed-cycle helium cryostat was used to study the above wafer samples and the fabricated devices, as a function of temperature. All spectra were measured using the conventional combination of a single-grating monochromator, InGaAs photodiode detector, and lock-in amplifier. ${ }^{15}$

\section{ROOM TEMPERATURE SURFACE REFLECTIVITY AND EDGE LUMINESCENCE}

The aforementioned fact that the VCSEL devices operated at RT tentatively suggests that the QW gain peak and the CM dip are aligned near the desired operating temperature of $\sim 300 \mathrm{~K}$. In fact, one would normally interpret this as meaning that the energy of the QW GST $\left(E_{Q W}\right)$ is at a slightly higher energy than that of the $\mathrm{CM}\left(E_{Q W}>E_{C M}\right)$ at RT, such that they will come into resonance at the slightly elevated temperatures that occur during $\mathrm{CW}$ operation due to self-heating. However, we shall see later that these initial conclusions are misleading.

Turning to the spectroscopic studies of the pre-fabrication VCSEL wafer; as is well known, the top-surface luminescence spectrum of a VCSEL wafer is not a useful characterisation probe since it essentially yields only a peak at the wavelength of the $\mathrm{CM}$, because appreciable light can escape from the top only in the vicinity of this dip in the reflectivity spectrum. However, one can sometimes gain more useful information by studying the emission from the side, through a cleaved edge of the wafer, which yields a spectrum essentially unperturbed by the DBR mirror structures. ${ }^{16}$ Unfortunately, measurements of the edge-photoluminescence spectra (not shown here) proved unhelpful as the emission was very weak and noisy. It was also extremely broad (full-width at half-maximum, FWHM $>150 \mathrm{meV}$ ) probably because of additional photoinduced luminescence from surface and interface states. Therefore, we instead investigated the edge emission electroluminescence (EL) using electrical excitation, as described in Ref. 16.

An example of the RT edge-EL is shown in Fig. 1. Here, the sample was biased in the forward direction with a voltage of $\sim 2.8 \mathrm{~V}$, and constant current pulses of $20 \mathrm{~mA}$, with a

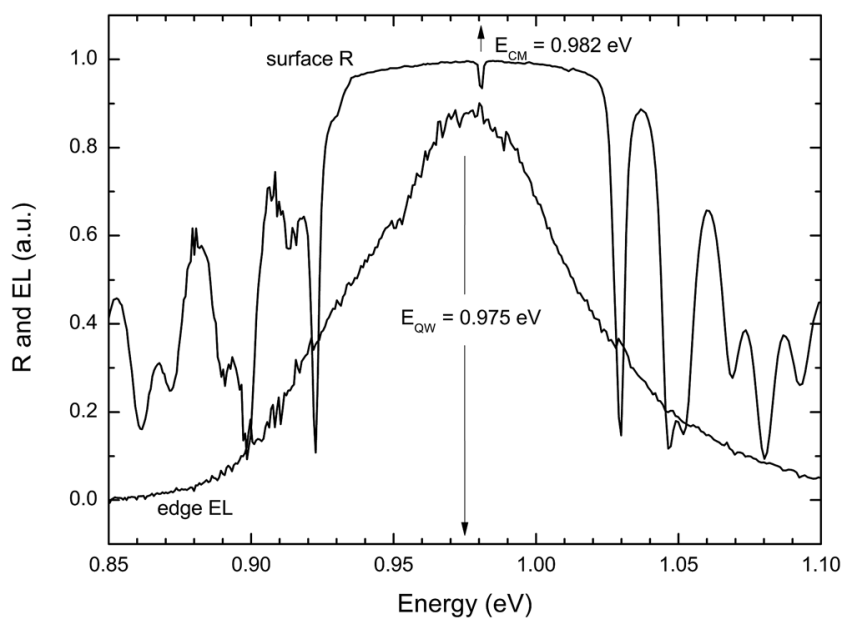

FIG. 1. Room temperature normal-incidence reflectivity $(R)$ spectrum of the GaAsSb VCSEL wafer sample, which shows that the position of the CM is near $E_{C M}=0.98 \mathrm{eV}$. High resolution scans indicate that the CM dip is very sharp with a FWHM $\sim 0.4 \mathrm{meV}$. The centre of the edge-emission EL peak is at a lower energy, implying that $E_{Q W}=0.975 \mathrm{eV}$ with a FWHM of $\sim 90 \mathrm{meV}$. This result that $E_{C M}>E_{Q W}$ at $\sim 300 \mathrm{~K}$ implies that the VCSEL device would need to be cooled for optimal performance. 
frequency of $15 \mathrm{~Hz}$ and a duty cycle of $1: 10$, to reduce heating effects. The resulting EL peak (which is near the QW GST energy $\left.E_{Q W}\right)$ is at $0.975 \pm 0.005 \mathrm{eV}(1272 \pm 7 \mathrm{~nm})$ and has a broad FWHM of $\Gamma_{\mathrm{QW}} \sim 90 \mathrm{meV}$. There may be several contributory factors resulting in this large observed EL width. First, the EL is due to the spontaneous emission of carriers in the QW and is naturally broad, particularly at high temperature. Second, the three QWs in the active region may have slight structural and compositional differences resulting in a spread of the associated EL peaks.

Also shown in Fig. 1 is the normalised front-surface reflectivity $(R)$ spectrum, which exhibits a CM dip energy $\left(E_{C M}\right)$ near $0.98 \mathrm{eV}$. Higher resolution $R$ scans indicated that the CM dip is at $0.9805 \pm 0.0002 \mathrm{eV}(1264.5 \pm 0.2 \mathrm{~nm})$ and is actually very sharp with a FWHM of $\Gamma_{C M} \sim 0.4 \mathrm{meV}$, corresponding to a finesse of more than 2400 . Comparing this with the above result for the width of EL peak, we see that $\Gamma_{Q W} \gg \Gamma_{C M}$. We will mention this important fact again in discussing the results of Sec. IV.

The comparison of EL and $R$ spectra in Fig. 1 actually shows that $E_{Q W}<E_{C M}$ at the desired operating temperature of $\sim 300 \mathrm{~K}$. This suggests that the VCSEL growth is actually slightly non-ideal. It would appear that the aforementioned RT device operation is only made possible due to the large broadening of the QW transition $\left(\Gamma_{\mathrm{QW}} \sim 90 \mathrm{meV}\right)$.

Note that edge-EL is necessarily a destructive technique, because of the need to cleave the VCSEL wafer, in contrast to the results we show next using modulated reflectance (MR). It is worth mentioning that these MR experiments were actually performed before the destructive EL experiments, but, for the sake of clarity we do not present the results in the order they were performed.

\section{TEMPERATURE DEPENDENT ER AND EL}

As the EL showed that $E_{Q W}<E_{C M}$ at RT, the next logical step was to cool the wafer sample to bring both into alignment; both energies increase with cooling but $E_{Q W}$ moves approximately five times faster than $E_{C M}$ (see later) so that they will cross. It has been shown that MR is the most sensitive technique to study the QW-CM alignment, giving well-resolved features due to the $\mathrm{CM}$ and, under certain circumstances, additional MR features due to the QW transitions, of much sharper resolution than is possible with photoluminescence. ${ }^{17,18}$ Photo-modulated reflectance is the preferred non-contact, non-destructive version of MR. However, due to the already deposited Pt electrodes, we chose to use the electro-modulated reflectance (ER) version of the MR technique in these temperature-dependent studies. We investigated the ER as a function of applied square-wave voltage amplitude and DC bias.

Figure 2 shows the temperature dependence of the normal incidence ER spectra in the region of the CM feature for the case of an applied square-wave voltage of amplitude $\pm 2 \mathrm{~V}$, zero DC bias, and frequency $333 \mathrm{~Hz}$. The first thing to note is that these ER spectra show only a single feature due to the CM dip; no extra features due to QW transitions are evident. The observation of separate QW and CM features in MR relies on a range of circumstances including the require-

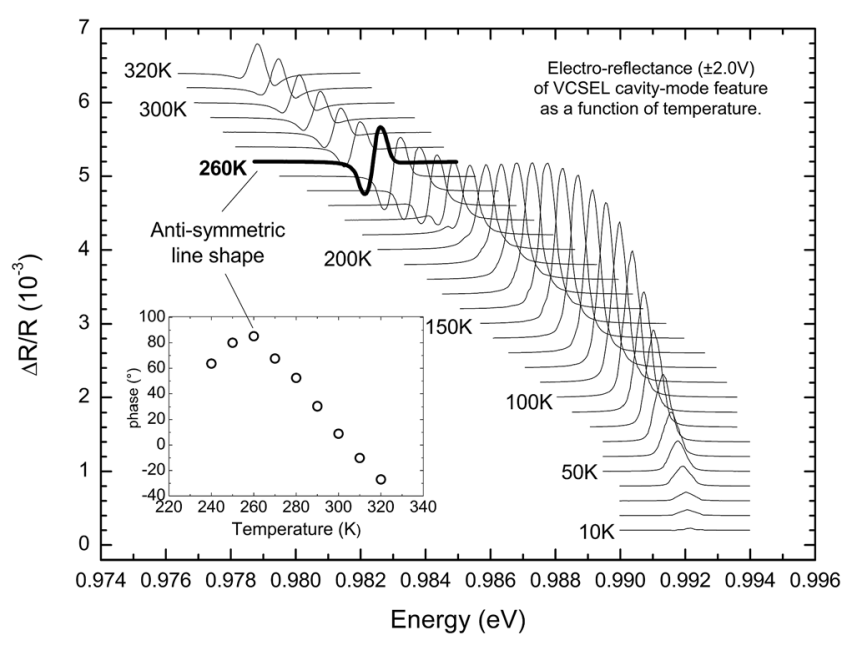

FIG. 2. Electro-reflectance (ER) signal in the vicinity of the VCSEL structure cavity-mode, as a function of temperature from 10 to $320 \mathrm{~K}$ in steps of $10 \mathrm{~K}$, as indicated to the left of the spectra (main plot). The inset shows the phase of the CM ER feature, obtained by fitting with an empirical Aspnes lineshape. This indicates that the feature is nearly perfectly anti-symmetrical (i.e., phase of $90^{\circ}$ ) close to $260 \mathrm{~K}$ (spectrum highlighted in the main plot), which indicates a crossing between the energies of the $\mathrm{CM}$ and QW ground state transition.

ment that the upper DBR must not have too high a reflectivity and that the QW and CM MR features must be of comparable broadening. ${ }^{17,18}$ These requirements are clearly not satisfied here, especially the latter since we concluded earlier that $\Gamma_{Q W} \gg \Gamma_{C M}$. However, this does not mean one cannot deduce information about the energy-position of the QW transitions as it is also known that one can infer when there is a CM-QW alignment by examining either the symmetry or the amplitude of the MR feature associated with the CM dip. ${ }^{17,18}$ Furthermore, it has been shown that in situations where $\Gamma_{Q W} \gg \Gamma_{C M}$ the amplitude of the $\mathrm{CM}$ feature is a quite misleading parameter, and rather it is the symmetry which one must examine. ${ }^{17,18}$ When a resonance occurs between a sharp CM dip and a much broader QW GST feature, a virtually perfect anti-symmetric MR line shape is predicted. ${ }^{17,18}$

Bearing this in mind, it is clear from a careful examination of Fig. 2 that the CM ER feature at RT $(300 \mathrm{~K})$ starts with a nearly symmetrical oscillatory line shape, and on cooling passes through an intermediate state at $\sim 260 \mathrm{~K}$ where the oscillator becomes almost perfectly antisymmetric, which by the above arguments indicates the occurrence of the resonance between the CM and QW GST features at this temperature. In order to quantify the degree of anti-symmetry of the ER features, they were fitted with the Aspnes third derivative functional form, ${ }^{19}$ which provides an empirical description of the symmetry of the line shape via its phase parameter. ${ }^{18}$ The inset shows the results for the fitted phase parameter, with a phase of $90^{\circ}$ indicating perfect anti-symmetry, showing a peak near $90^{\circ}$ occurring at $260 \pm 5 \mathrm{~K}$, as expected.

Upon further cooling, we observe that the CM ER signal becomes more symmetrical again, but, unlike the high temperature spectra, this time the profile is positive-definite (i.e., lacking any significant negative-going oscillations). Meanwhile, its amplitude increases rapidly with cooling, reaching 
a maximum at $\sim 120 \mathrm{~K}$, after which it drops rapidly (by about two orders of magnitude) while maintaining a roughly symmetrical positive-definite profile. The implications of these lineshape changes are discussed in detail next.

As explained above, the peak in amplitude is of no significance in interpreting any CM-QW resonances but it is helpful here to discuss further the behaviour of the CM ER line shapes observed in Fig. 2. Here, we give only qualitative arguments and refer the reader to the seminal papers for a more detailed mathematical understanding. ${ }^{17,18}$ A modulated reflectivity spectrum can be written in general as ${ }^{19}$

$$
\Delta R / R=\alpha \Delta \varepsilon_{1}+\beta \Delta \varepsilon_{2},
$$

where $\alpha$ and $\beta$ are the Seraphin coefficients and $\Delta \varepsilon_{1}$ and $\Delta \varepsilon_{2}$ the real and imaginary parts of the modulated dielectric function, respectively.

It has been shown that, in the case of a VCSEL in the vicinity of $E_{C M}, \alpha$ and $\beta$ can be essentially assumed to result from the modulation of the refractive index and absorption, respectively, of the active cavity region, while, in the vicinity of the QW GST, $\Delta \varepsilon_{1}$ and $\Delta \varepsilon_{2}$ result from the modulated complex dielectric function of the QW. ${ }^{17}$ Both pairs of parameters form Kramers-Kronig couples so that, while $\alpha$ has a dispersive (i.e., anti-symmetric) shape, $\beta$ has an absorptive (symmetric) form—see Fig. 3(a) (left and right, respectively). In contrast, $\Delta \varepsilon_{1}$ has an essentially absorptive (symmetric) form while $\Delta \varepsilon_{2}$ is dispersive (anti-symmetric) — see Fig. 3(b). However, in the present situation where $\Gamma_{Q W} \gg \Gamma_{C M}$, then, when the $\mathrm{CM}$ and QW energies are in close proximity and the MR features overlap, both products $\alpha \Delta \varepsilon_{1}$ and $\beta \Delta \varepsilon_{2}$ are

.
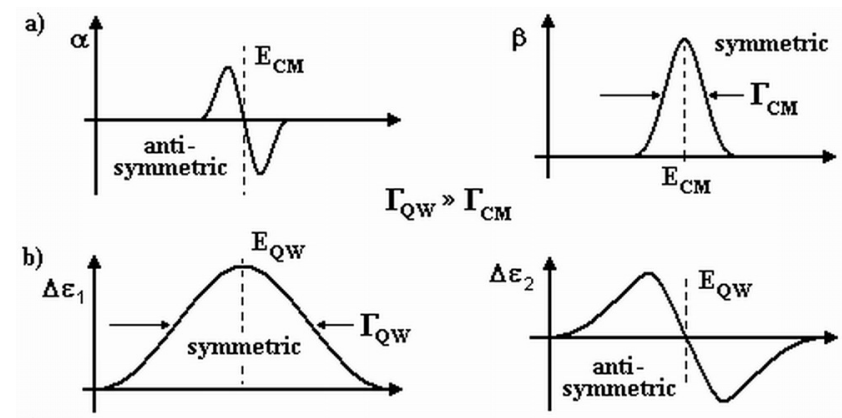$$
\text { c) }
$$

$\alpha \cdot \Delta$
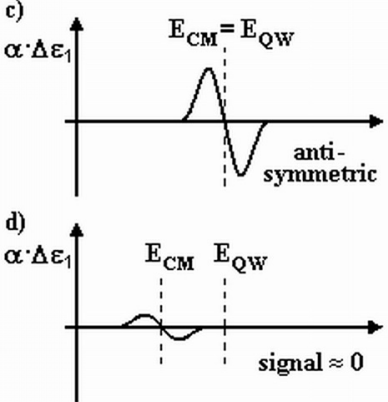
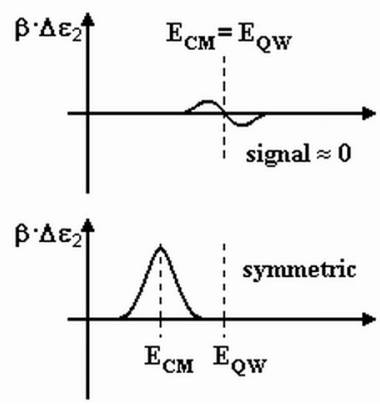

FIG. 3. Schematic lineshapes of: (a) the Seraphin coefficients $\alpha$ and $\beta$ in the vicinity of the $\mathrm{CM}$ energy $\left(E_{C M}\right)$ and; (b) of the modulated dielectric function $\left(\Delta \varepsilon_{1}\right.$ and $\left.\Delta \varepsilon_{2}\right)$ in the vicinity of the QW ground-state transition energy $\left(E_{Q W}\right)$, in the situation where the broadening of the $\mathrm{QW}$ feature is much larger than that of the CM. (c) shows their respective products $\alpha \Delta \varepsilon_{1}, \beta \Delta \varepsilon_{2}$ near resonance, i.e., $E_{C M} \approx E_{Q W}$. (d) shows the products when away from resonance, e.g., when $E_{C M}<E_{Q W}$.

essentially anti-symmetric in nature and have spectral profiles with symmetries determined primarily by those of the Seraphin coefficients. This is illustrated qualitatively in Fig. 3(c), left and right, respectively.

Note also from the example situation in Fig. 3(c), that in the situation where $E_{C M}=E_{Q W}$ the $\beta \Delta \varepsilon_{2}$ term actually becomes very small compared to $\alpha \Delta \varepsilon_{1}$, because $\Delta \varepsilon_{2}$ is weak and broad and slowly crossing zero at $\sim E_{Q W}$, while, though $\Delta \varepsilon_{1}$ is also weak and broad, it is maximal at $E_{Q W}$. Thus, the $\alpha \Delta \varepsilon_{1}$ term is actually dominant, resulting in a predicted line shape that is virtually perfectly anti-symmetric when $E_{C M}=E_{Q W}$.

As one moves slightly away from the resonance condition, for instance due to changing the temperature (see Figs. $3(\mathrm{~d}))$, the amplitude of the $\beta \Delta \varepsilon_{2}$ product may temporarily increase significantly, and, because $E_{Q W}$ now lies off-centre from $E_{C M}$, the $\beta \Delta \varepsilon_{2}$ product can yield a roughly symmetric line shape of quite large amplitude. On the other hand, the $\alpha \Delta \varepsilon_{1}$ product is still approximately anti-symmetrical, but its amplitude is strongly reduced in this "de-tuned" non-resonance condition. The net amplitude of the $\Delta R / R$ signal in this case is given by the sum according to Eq. (1), but, since the broad $\Delta \varepsilon_{1}$ and $\Delta \varepsilon_{2}$ profiles are approximately constant over the region of the sharp CM feature, the $\Delta R / R$ signal is given essentially by a linear combination of the Seraphin coefficients. Since the respective magnitudes and widths of $\alpha$ and $\beta$ depend on structural details of the particular sample, the amplitude of the $\Delta R / R$ signal cannot be used as a reliable indicator of any resonance condition in these conditions. In our case, it is clear from Fig. 2 that, as we cool below the resonance condition temperature, the product $\beta \Delta \varepsilon_{2}$ is actually stronger than the $\alpha \Delta \varepsilon_{1}$ component, so the observed ER amplitude increases and reaches a symmetrically shaped maximum at $\sim 120 \mathrm{~K}$ (Fig. 2). However, as explained earlier, this is an artefact and cannot be used to infer any information of use for our present purposes. Furthermore, the observed sign of this $\beta \Delta \varepsilon_{2}$ signal is of no significance and depends on the phase settings of the lock-in amplifier.

Further cooling causes $E_{C M}$ and $E_{Q W}$ to shift even further apart and one can see from Fig. 3 that it is inevitable that the overall $\Delta R / R$ magnitude must eventually decrease to very small values as the respective $C M$ and $Q W$ features move increasingly further out of each other's influence. The final drop in ER amplitude seen in Fig. 2 towards $10 \mathrm{~K}$ may also be partly attributed to the freeze-out of the carriers in the doped DBRs and thus an increase of the width of the depletion region. Hence, the constant external modulation voltage would then result in a smaller field modulation of the actual QW region, so that the ER signal drops away.

To provide further confirmation of the CM/QW GST ER resonance found at $\sim 260 \mathrm{~K}$, we performed edge EL measurements similar to that shown in Fig. 1, but as a function of temperature. The resulting peak positions of the edge EL are plotted in Fig. 4 (filled squares) together with the CM positions obtained from normalised normal-incidence $R$ measurements (open triangles), which were obtained simultaneously with the ER measurements. The large error bars on the EL peaks are due to the very broad EL spectra. The corresponding uncertainties in the $\mathrm{CM}$ energies are within the symbol 


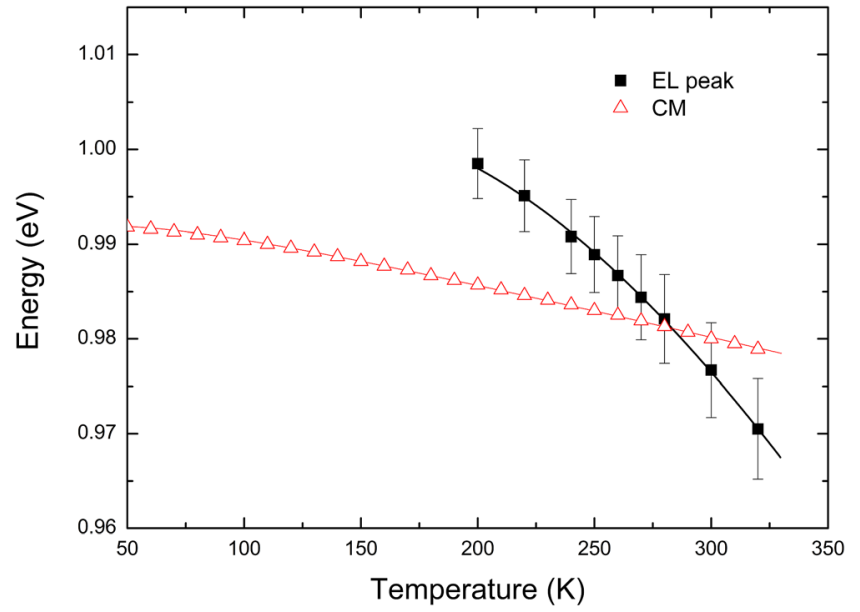

FIG. 4. Edge electro-luminescence (EL) peak positions (filled squares) and $\mathrm{CM}$ positions from normalised normal-incidence reflectivity measurements (open triangles) as a function of temperature. The curves, which are only guides to the eye, cross at $280 \mathrm{~K} \pm 20 \mathrm{~K}$, thus confirming the ER result of Fig. 2.

size of Fig. 4. We observe a crossing of the two data sets at about $280 \pm 20 \mathrm{~K}$ in Fig. 4, which, though rather approximate, is equal to within experimental uncertainty to the resonance temperature $260 \pm 5 \mathrm{~K}$ found in the ER of Fig. 2. Apart from the rather large uncertainty due to the broad EL peaks, the difference in the two deduced tuning temperatures may possibly also be due to the different applied electric field amplitude in the two experiments; while the ER was performed in an unbiased configuration, thus resulting in only small applied internal fields, the EL experiments were performed with a forward bias, which, due to the injection of carriers, screens any built-in internal fields. Thus, in the EL experiments the small red-shift in the transition energy induced via the quantum confined Stark effect ${ }^{20}$ is slightly reduced and would result in a slightly higher $E_{Q W}$ transition energy. This would then give a slightly higher crossover temperature in the EL results of Fig. 4 as compared to the ER results in Fig. 2.

From the two sets of spectroscopic results in Figs. 2 and 4, we may conclude reasonably confidently that the CM and QW GST are in resonance at a temperature of $270 \pm 10 \mathrm{~K}$ in this VCSEL wafer.

\section{DEVICE MEASUREMENTS-SHIFT OF LASING WAVELENGTH WITH TEMPERATURE}

We now return to the aforementioned studies on devices fabricated from the VCSEL wafer. An EEL was also grown and fabricated with nominally the same active region as the VCSEL. The cladding in the EEL is $\mathrm{Al}_{0.65} \mathrm{Ga}_{0.35} \mathrm{As}$. The length of the fabricated EEL is $1000 \mu \mathrm{m}$ with a $50 \mu \mathrm{m}$-width ridge stripe. Fig. 5 shows the temperature dependence of the lasing emission energies of the two devices, both under pulsed operating conditions of 500 ns-long pulses at a duty cycle of $10 \mathrm{kHz}$ (for the VCSEL) and $100 \mathrm{~Hz}$ (for the EEL). The VCSEL device diameter in these studies was $9 \mu \mathrm{m}$. The different duty cycles were used in order to maximise the strength of the measured integrated signal whilst minimising Joule heating. The weaker luminescence from the VCSEL

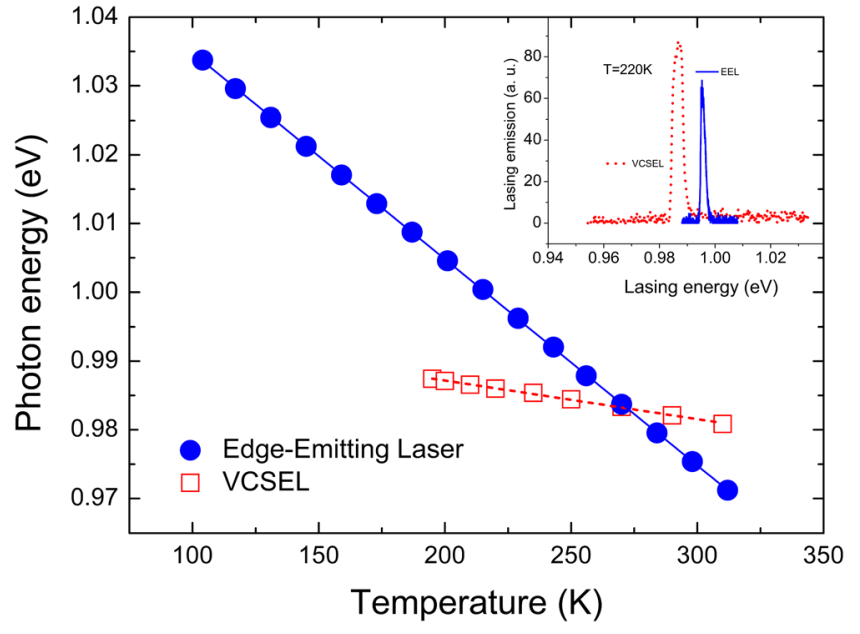

FIG. 5. Shift of the pulsed lasing photon energy of the VCSEL (open squares) together with the corresponding shift of the edge emitter (filled circles) with nominally identical active region structure. The cross-over is at $267 \mathrm{~K}$, which confirms the earlier spectroscopic results about the CM/QW alignment. The fitted straight lines show that both behaviours are approximately linear over the studied temperature range. The inset shows difference in lasing peaks between the EEL and VCSEL at $220 \mathrm{~K}$.

(due to the smaller emitting area) and lower absolute threshold current meant that a higher repetition rate was needed for the VCSEL measurements. The pulse width was fixed to avoid increasing the heating during the pulse. Thus, for improved signal (i.e., signal/noise ratio) the VCSEL was operated at a higher frequency.

The shift of the EEL emission energy reflects the temperature dependence of the gain peak and thus that of the emission peak due to the QW GST, which in turn is given by the thermally induced shift in bandgap energy. In this temperature range (between $\sim 100 \mathrm{~K}$ and $310 \mathrm{~K}$ ), the shift is approximately linear and can be fitted with

$$
\mathrm{E}_{\mathrm{EEL}}(T)=1.0648 \mathrm{eV}-\mathrm{T} \times 0.300 \mathrm{meV} / \mathrm{K} .
$$

This is comparable to the corresponding shift in the edgeEL peaks seen in Fig. 4 which can be fitted with the straight line

$$
\mathrm{E}_{\text {edge-EL }}(\mathrm{T})=1.0461 \mathrm{eV}-\mathrm{T} \times 0.231 \mathrm{meV} / \mathrm{K} .
$$

These two fitted lines are equal to within the experimental uncertainties in the edge-EL energies (see Fig. 4).

The shift of the VCSEL emission with temperature is determined only by the shift of the CM dip which is much slower than the shift of the QW GST, and is due only to thermal changes in the cavity thickness and refractive index. ${ }^{21}$ This is also approximately linear in this temperature range and can be fitted with

$$
\mathrm{E}_{\mathrm{VCSEL}}(\mathrm{T})=0.9984 \mathrm{eV}-\mathrm{T} \times 0.056 \mathrm{meV} / \mathrm{K} .
$$

This matches quite well with the shift of the CM in Fig. 4 measured from the reflectivity measurements on the prefabrication wafer, which, over the same temperature range as Fig. 5 can also be satisfactorily fitted with a straight line

$$
\mathrm{E}_{\mathrm{CM}}(\mathrm{T})=0.9969 \mathrm{eV}-\mathrm{T} \times 0.056 \mathrm{meV} / \mathrm{K}
$$


The slight bodily blue-shift of $\sim 1.5 \mathrm{meV}$ of the VCSEL fitted line (Eq. (4)) with respect to the CM fitted line (Eq. (5)) is most probably due to slight differences in cavity thickness between the fabrication and pre-fabrication regions of the wafer. The cross-over of the two straight lines fitted to the device results in Fig. 5 (Eqs. (2) and (4)), and thus the CM-QW alignment deduced from that, is found to occur at $\mathrm{T}=272 \pm 5 \mathrm{~K}$ which agrees very well with the earlier wafer spectroscopy result of $270 \pm 10 \mathrm{~K}$.

These observations have consequences regarding the device performance; at the operating current the gain peak has to be well aligned with cavity mode dip in an operating VCSEL. If the CM and QW are not tuned at the desired operating temperature, this will necessitate higher threshold currents to enable lasing. In a recent separate set of temperature-dependent studies of the spontaneous and lasing emission characteristics of the EEL and VCSEL devices of Fig. 5, we showed that the radiative component of the threshold current is indeed minimal at the CM-QW tuning temperature of $\sim 270 \mathrm{~K}$ deduced here from the spectroscopic studies. $^{22}$

In the absence of any other temperature dependencies, the total threshold current will also be a minimum at this temperature. However, it is important to note that there are also highly temperature-dependent non-radiative contributions to the threshold current and that these compete with the CM-QW tuning effect; with, say heating from low temperature, while the CM and QW are moving into better alignment, so producing a steady drop in the radiative component of the threshold current, the non-radiative losses are increasing and divert ever-increasing proportions of the total driving current into deleterious non-radiative recombination as the device is warmed. The end result is a "trade-off" between these two effects and so that the threshold current in the VCSEL device is actually at a minimum at a somewhat lower temperature $(\sim 225 \mathrm{~K})$ than the CM-QW tuning temperature deduced here from the purely spectroscopic studies. These effects are described in full detail in Ref. 22 and must be considered when optimising a VCSEL design.

\section{SUMMARY AND CONCLUSIONS}

We have shown that a GaAs-based VCSEL with GaAsSb QWs operates near the silica-fibre $1.31 \mu \mathrm{m}$ zero dispersion wavelength in $\mathrm{CW}$ mode at $300 \mathrm{~K}$, important for metropolitan-area fibre-to-the-home networks. Nondestructive ER and destructive edge-EL spectroscopic measurements on pre-fabrication wafer samples show that the growth has resulted in a slight misalignment between the $\mathrm{CM}$ dip and the QW ground-state transition energies $\left(E_{Q W}<E_{C M}\right)$ at $300 \mathrm{~K}$ and only come into the "tuned" state $\left(E_{Q W}=E_{C M}\right)$ at the lower temperature of $270 \pm 10 \mathrm{~K}$. The results also indicate that the aforementioned CW VCSEL lasing observed at $300 \mathrm{~K}$ occurs because of the large broadening of the QW emission, which is approximately 200 times wider than the CM dip in the VCSEL. We have explained how this great difference in broadening means that the ER spectral line shape becomes anti-symmetrical at the tuning temperature. This effect has in fact been used to determine the tuning temperature, by quantifying the degree of antisymmetry of the ER line shape as a function of temperature. The spectral emission peaks of fabricated VCSEL devices have also been studied as a function of temperature and compared with those of EELs with nominally identical active regions to those of the VCSEL, showing that the EEL and VCSEL emission wavelengths cross at the same tuning temperature as determined by the ER and EL studies on the prefabrication wafer samples. We have discussed how these results indicate that the radiative component of the threshold current should be minimal near $270 \mathrm{~K}$. Finally, the results reported here are discussed in light of our separate study of the temperature dependence of the actual threshold current in operating VCSEL devices, where the contribution of competing non-radiative recombination processes is examined in detail. The techniques used here provide a useful insight into the factors influencing the operating characteristics of the VCSELs and provide a means of further device optimisation. The utility of the non-destructive ER characterisation technique has been clearly demonstrated for these VCSEL structures.

\section{ACKNOWLEDGMENTS}

We acknowledge support from the EPSRC(UK) under grants GR/T21516/01 and EP/H005587/1. TJCH thanks the "Malaysian University Grant Program Tier 1," Universiti Teknologi Malaysia (Q.J130000.7126.01H55) and M.O.H.E for funding.

${ }^{1}$ A. J. Mayhew, S. J. Page, A. M. Walker, and S. I. Fisher, BT Technol. J. 20, 91 (2002).

${ }^{2}$ Y.-F. Lao, C.-F. Cao, H.-Z. Wu, M. Cao, and Q. Gong, Electron. Lett. 45(2), 105 (2009).

${ }^{3}$ S. J. Sweeney, D. McConville, N. F. Massé, R.-X. Bouyssou, A. R. Adams, C. N. Ahmad, and C. Hanke, Phys. Status Solidi B 241(14), 3391-3398 (2004).

${ }^{4}$ O. B. Shchekin and D. G. Deppe, IEEE Photon. Technol. Lett. 14, 1231 (2002).

${ }^{5}$ I. P. Marko, A. R. Adams, S. J. Sweeney, D. J. Mowbray, M. S. Skolnik, H. Y. Liu, and K. M. Groom, IEEE J. Sel. Top. Quantum Electron. 11(5), 1041 (2005).

${ }^{6}$ M. Yamada, T. Anan, K. Kurihara, K. Nishi, K. Tokutome, A. Kamei, and S. Sugou, Electron. Lett. 36, 637 (2000).

${ }^{7}$ P. Dowd, W. Braun, C. M. Ryu, C.-Z. Guo, S. L. Chen, U. Koelle, S. R. Johnson, and Y.-H. Zhang, CLEO 1999 Tech. Dig. (IEEE Cat. No.99CH37013), 1999, 489.

${ }^{8}$ N. Samal, Y. Cao, D. Ding, J.-B. Wang, S.-Q. Yu, X. Jin, S. A. Chaparro, Yu. G. Sadofyev, S. R. Johnson, and Y.-H. Zhang, in SPIE Photonics West, San Jose, CA, January 2003.

${ }^{9}$ S.-Q. Yu, D. Ding, J.-B. Wang, N. Samal, X. Jin, Y. Cao, S. R. Johnson, and Y.-H. Zhang, J. Vac. Sci. Technol. B 25(5), 1658 (2007).

${ }^{10}$ P. Dowd, S. R. Johnson, S. A. Feld, M. Adamcyk, S. A. Chaparro, J. Joseph, K. Hilgers, M. P. Horning, K. Shiralagi, and Y.-H. Zhang, Electron. Lett. 39, 987 (2003).

${ }^{11}$ X. Jin, S.-Q. Yu, Y. Cao, D. Ding, J.-B. Wang, N. Samal, Yu. G. Sadofyev, S. R. Johnson, and Y.-H. Zhang, in Proceedings of the IEEE Lasers and Electro-Optics Society 16th Annual Meeting (IEEE Cat. No.03CH37460) (2003), Vol. 1, p. 69.

${ }^{12}$ Yu. G. Sadofyev, S. R. Johnson, S. A. Chaparro, Y. Cao, D. Ding, J.-B. Wang, K. Franzreb, and Y.-H. Zhang, Appl. Phys. Lett. 84, 3546 (2004).

${ }^{13}$ M. Zujewski and W. Nakwaski, Opto-Electron. Rev. 18(2), 181 (2010).

${ }^{14}$ S. R. Johnson, C. Z. Guo, S. Chaparro, Yu. G. Sadofyev, J. Wang, Y. Cao, N. Samal, J. Xu, S. Q. Yu, D. Ding, and Y.-H. Zhang, J. Cryst. Growth 251, 521 (2003).

${ }^{15}$ T. K. Sharma and T. J. C. Hosea, Jpn. J. Appl. Phys. 48, 082301 (2009). 
${ }^{16}$ S. Ghosh, S. Constant, T. J. C. Hosea, and T. E. Sale, J. Appl. Phys. 88, 1432 (2000).

${ }^{17}$ T. J. C. Hosea, Thin Solid Films 450, 3 (2004).

${ }^{18}$ S. Ghosh, S. B. Constant, and T. J. C. Hosea, Appl. Phys. Lett. 78, 3250-3252 (2001).

${ }^{19}$ D. E. Aspnes, Surf. Sci. 37, 418 (1973).
${ }^{20}$ S. Schmitt-Rink, D. S. Chemla, and D. A. B. Miller, Adv. Phys. 38, 89 (1989).

${ }^{21}$ G. Knowles, S. J. Sweeney, T. E. Sale, and A. R. Adams, Phys. Status Solidi B 223, 581 (2001).

${ }^{22}$ K. Hild, I. P. Marko, S. R. Johnson, S.-Q. Yu, Y.-H. Zhang, and S. J. Sweeney, Appl. Phys. Lett. 99, 071110 (2011). 